\title{
Corneal Touch
}

National Cancer Institute

\section{Source}

National Cancer Institute. Corneal Touch. NCI Thesaurus. Code C50513.

Complications of intraocular lens surgery; usually refers to intraocular lens contact with cornea, can be intermittent or chronic. 